\title{
THE GROWTH OF SOLUTIONS OF SECOND ORDER LINEAR DIFFERENTIAL EQUATIONS WITH MEROMORPHIC COEFFICIENTS*
}

\author{
ZONG-XUAN CHEN
}

\begin{abstract}
In this paper, we investigate the growth of infinite order meromorphic solutions of second order differential equations with transcendental meromorphic coefficients. For most of meromorphic solutions, we obtain some precise estimates of their hyper-order.
\end{abstract}

\section{Introduction and results}

Throughout the presentation, we use the standard notations of the Nevanlinna theory (e.g. see $[10,13])$. In addition, we use notations $\lambda(f)$ and $\lambda(1 / f)$ to denote respectively the exponent of convergence of the zeros and the poles of meromorphic function $f(z), \sigma(f)$ to denote the order of growth of $f(z)$ and $\mu(f)$ to denote the lower order of $f(z)$. In order to express the rate of growth of meromorphic function of infinite order, we recall the following definition (e.g. see [18]).

DeFINITION 1. Let $f$ be a meromorphic function, then we define the hyperorder $\sigma_{2}(f)$ of $f(z)$

$$
\sigma_{2}(f)=\varlimsup_{r \rightarrow \infty} \frac{\log ^{+} \log ^{+} T(r, f)}{\log r} .
$$

For the second order linear differential equation

$$
f^{\prime \prime}+A(z) f^{\prime}+B(z) f=0,
$$

it is well known that let $A(z)$ or $B(z)$ be transcendental, if $f_{1}$ and $f_{2}$ are two linearly independent meromorphic solutions of (1.1) then by [7, Lemma 3], there is at least one of $f_{1}, f_{2}$ such that it's order is infinite. In [12], S. Hellerstein, J. Miles, and J. Rossi proved that if $A(z)$ and $B(z)$ are entire functions with $\sigma(B)<\sigma(A) \leq 1 / 2$, then any nonconstant solution of (1.1) has infinite order.

Key words: differential equation, meromorphic function, hyper-order

AMS No: 34A20, 30D35

* The work was supported by the National Natural Science Foundation of China

Received February 26, 1998; revised November 10, 1998. 
GROWTH OF SOLUTIONS OF SECOND ORDER LINEAR DIFFERENTIAL EQUATIONS 209

For the case where $\sigma(B)<\sigma(A)<1 / 2$, the above assertion was proved in [9] by $\mathrm{G}$. Gundersen. For higher order linear differential equations with entire coefficients of small growth, J. K. Langley investigated the zeros of infinite order solutions in [17]. Then for a great deal of meromorphic solutions with infinite order, more precise estimates for their rate of growth is a very important aspect. Ki-Ho Kwon investigated the problem and obtained the following result in $[15]$ :

THEOREM A. Let $A(z)$ and $B(z)$ be entire functions such that $\sigma(A)<\sigma(B)$ or $\sigma(B)<\sigma(A)<1 / 2$. Then every solution $f(\not \equiv 0)$ of $(1.1)$ satisfies $\sigma_{2}(f) \geq$ $\max \{\sigma(A), \sigma(B)\}$.

In [16], Ki-Ho Kwon investigated the hyper-order $\sigma_{2}(f)$ of solutions of (1.1) where $A=h_{1}(z) e^{P(z)}, B=h_{2}(z) e^{Q(z)}, P(z), Q(z)$ are polynomials satisfying $\operatorname{deg} P$ $=\operatorname{deg} Q, h_{1}, h_{2}$ are entire functions satisfying $\sigma\left(h_{j}\right)<\operatorname{deg} P$. In [5] Zong-Xuan Chen and Chung-Chun Yang investigated the hyper-order of infinite order entire solutions of higher order linear differential equation with entire coefficients.

In this paper we will consider the hyper-order of meromorphic solutions of the general differential equations with meromorphic coefficients. It is easy to see that most of meromorphic solutions of (1.1) satisfy

$$
\varlimsup_{r \rightarrow \infty} \frac{\log \log N(r, 1 / f)}{\log r} \leq \max \{\sigma(A), \sigma(B)\} .
$$

For the solution satisfying (1.2), we obtain the more precise result $\sigma_{2}(f)=$ $\max \{\sigma(A), \sigma(B)\}$ than $\sigma_{2}(f) \geq \max \{\sigma(A), \sigma(B)\}$ in Theorem A.

THEOREM 1. Let $A(z)$ and $B(z)$ be meromorphic functions such that

$$
\max \{\sigma(A), \lambda(1 / B)\}<\sigma(B)<+\infty .
$$

If the equation (1.1) has meromorphic solutions, then every meromorphic solution $f(\not \equiv 0)$ satisfies $\sigma_{2}(f) \geq \sigma(B)$.

Furthermore, if $f$ satisfies

$$
\varlimsup_{r \rightarrow \infty} \frac{\log \log N(r, 1 / f)}{\log r} \leq \sigma(B)
$$

then $\sigma_{2}(f)=\sigma(B)$.

THEOREM 2. Let $A(z)$ and $B(z)(\not \equiv 0)$ be meromorphic functions such that

$$
\max \{\sigma(B), \lambda(1 / A)\}<\mu(A) \leq \sigma(A)<1 / 2 .
$$

If the equation (1.1) has meromorphic solutions, then every meromorphic solution $f(\not \equiv 0)$ satisfies $\sigma_{2}(f) \geq \sigma(A)$. 
Furthermore, if $f$ satisfies

$$
\varlimsup_{r \rightarrow \infty} \frac{\log \log N(r, 1 / f)}{\log r} \leq \sigma(A)
$$

then $\sigma_{2}(f)=\sigma(A)$.

\section{Lemmas that are needed for the proof of Theorem 1}

LemMa 1 ([8, Theorem 4]). Let $f$ be a meromorphic function, and let $\alpha>1$ and $\varepsilon>0$ be given real constants. Then there exists a set $E_{1} \subset(0, \infty)$ that has finite linear measure and there exists constant $B>0$ that depend only on $\alpha$, such that for all $z$ satisfying $|z|=r \notin E_{1}$, we have

$$
\left|\frac{f^{(j)}(z)}{f(z)}\right| \leq B\left(T(\alpha r, f) r^{\varepsilon} \log T(\alpha r, f)\right)^{j}, \quad(j=1,2) .
$$

LEMMA 2 ([15]). Let $g(z)$ be a nonconstant entire function of finite order. Then for any given $\varepsilon>0$, there exists a set $H_{2} \subset(0, \infty)$ with $\overline{\text { dens }} H_{2}=1$ such that

$$
M(r, g) \geq \exp \left\{r^{\sigma(g)-\varepsilon}\right\}
$$

for all $r \in H_{2}$.

For a set $H \subset[0, \infty)$, the upper and the lower densities of the $H$ are defined by

$$
\overline{\operatorname{den} s} H=\varlimsup_{r \rightarrow \infty} \frac{m(H \cap[0, r])}{r}
$$

and

$$
\underline{\text { dens }} H=\varliminf_{r \rightarrow \infty} \frac{m(H \cap[0, r])}{r}
$$

where $m(F)$ is the linear measure of a set $F$.

LEMMA 3. Let $w(z)$ be a meromorphic function with $\lambda(1 / w)<\sigma(w)<+\infty$. Then for any given $\varepsilon>0$, there exists a set $H_{3} \subset[0,+\infty)$ with $\overline{\text { dens }} H_{3}=1$ such that

$$
M(r, w) \geq \exp \left\{r^{\sigma(w)-\varepsilon}\right\}
$$

holds for all $r \in \mathrm{H}_{3}$.

Proof. Set $w(z)=z^{k} g(z) / d(z)$, where $k$ is an integer, $g(z)$ is an entire function, $d(z)$ is the canonical product (or polynomial) formed with the nonzero poles of $w(z)$, hence $\lambda(d)=\sigma(d)=\lambda(1 / w)<\sigma(w)$ and $\sigma(g)=\sigma(w)$. By Lemma 2 , for any given $\varepsilon(0<2 \varepsilon<\sigma(w)-\lambda(1 / w))$, there exists a set $H_{2} \subset[0,+\infty)$ with 
GROWTH OF SOLUTIONS OF SECOND ORDER LINEAR DIFFERENTIAL EQUATIONS 211 $\overline{\text { dens }} H_{2}=1$ such that

$$
M(r, g) \geq \exp \left\{r^{\sigma(w)-\varepsilon}\right\}, \quad\left(r \in H_{2}\right) .
$$

Now take a point $z_{r}$ satisfying $\left|z_{r}\right|=r \in H_{2}$ and $\left|g\left(z_{r}\right)\right|=M(r, g)$. Since there is $R(>0)$ such that for $r>0$, we have

$$
\left|d\left(z_{r}\right)\right| \leq \exp \left\{r^{\lambda(1 / w)+\varepsilon}\right\} .
$$

Set an $H_{3}=H_{2}-[0, R]$, then $\overline{d e n s} H_{3}=1$. By (2.4) and (2.5),

$$
M(r, w) \geq\left|z_{r}^{k} g\left(z_{r}\right) / d\left(z_{r}\right)\right| \geq \exp \left\{r^{\sigma(w)-\varepsilon}\right\} .
$$

holds for $\left|z_{r}\right|=r \in H_{3}$.

LEMMA 4. Let $F(r), G(r)$ are nondecreasing functions on $[0, \infty)$ such that $F(r) \leq G(r)$ for $r \notin E_{4}$, where the set $E_{4} \subseteq[0, \infty)$ that has finite linear measure. Then

$$
\begin{gathered}
\varlimsup_{r \rightarrow \infty} \frac{\log F(r)}{\log r} \leq \varlimsup_{r \rightarrow \infty} \frac{\log G(r)}{\log r}, \\
\varlimsup_{r \rightarrow \infty} \frac{\log \log F(r)}{\log r} \leq \varlimsup_{r \rightarrow \infty} \frac{\log \log G(r)}{\log r} .
\end{gathered}
$$

Proof. Set the linear measure of the $E_{4}, m E_{4}=\delta<+\infty$. For any given sequence $\left\{r_{n}\right\} \subset[0,+\infty)\left(2 \delta<r_{1}<r_{2}<\cdots, r_{n} \rightarrow \infty\right)$, there exists a point $r_{n}^{\prime} \in$ $\left[r_{n}, r_{n}+2 \delta\right]-E_{4}$. From

$$
\frac{\log F\left(r_{n}\right)}{\log r_{n}} \leq \frac{\log F\left(r_{n}^{\prime}\right)}{\log \left(r_{n}^{\prime}-2 \delta\right)} \leq \frac{\log G\left(r_{n}^{\prime}\right)}{\log r_{n}^{\prime}+\log \left(1-\left(2 \delta / r_{n}^{\prime}\right)\right)}
$$

we have

$$
\begin{aligned}
\varlimsup_{n \rightarrow \infty} \frac{\log F\left(r_{n}\right)}{\log r_{n}} & \leq \varlimsup_{n \rightarrow \infty} \frac{\log G\left(r_{n}^{\prime}\right)}{\log r_{n}^{\prime}+\log \left(1-\left(2 \delta / r_{n}^{\prime}\right)\right)} \\
& =\varlimsup_{n \rightarrow \infty} \frac{\log G\left(r_{n}^{\prime}\right)}{\log r_{n}^{\prime}} \leq \varlimsup_{r \rightarrow \infty} \frac{\log G(r)}{\log r}
\end{aligned}
$$

Since the sequence $\left\{r_{n}\right\} \subset[0,+\infty)$ is arbitrary, we have

$$
\varlimsup_{r \rightarrow \infty} \frac{\log F(r)}{\log r} \leq \varlimsup_{r \rightarrow \infty} \frac{\log G(r)}{\log r} .
$$

Similarly, we have

$$
\varlimsup_{r \rightarrow \infty} \frac{\log \log F(r)}{\log r} \leq \varlimsup_{r \rightarrow \infty} \frac{\log \log G(r)}{\log r}
$$


Lemma 5 (see [6]). Let $f(z)$ is a meromorphic function, $f(0) \neq \infty$. Then for $\tau>1$ and $r>0$, we have

$$
T(r, f)<C_{\tau} T\left(\tau r, f^{\prime}\right)+\log ^{+}(\tau r)+4+\log ^{+}|f(0)|,
$$

where $C_{\tau}(>0)$ is a constant depending only on $\tau$.

LEMMA 6 ([14, Theorem 12.4]). Let $f$ be an entire function with $\sigma(f)=\infty$. Then $f$ can be represented in the form $f(z)=g(z) e^{h(z)}$ where $g(z)$ and $h(z)$ are entire functions such that

$$
\begin{aligned}
\sigma_{2}(f) & =\max \left\{\sigma_{2}(g), \sigma_{2}\left(e^{h}\right)\right\} \\
\sigma_{2}(g) & =\varlimsup_{r \rightarrow \infty} \frac{\log \log N(r, 1 / g)}{\log r}=\varlimsup_{r \rightarrow \infty} \frac{\log \log N(r, 1 / f)}{\log r} .
\end{aligned}
$$

LEMMA 7. Let $A(z), B(z)$ be meromorphic functions of finite order. If $f(z)$ is a meromorphic solution of (1.1) and satisfies

$$
\varlimsup_{r \rightarrow \infty} \frac{\log \log N(r, 1 / f)}{\log r} \leq \max \{\sigma(A), \sigma(B)\}=\sigma
$$

then $\sigma_{2}(f) \leq \sigma$.

Proof. Set $f(z)=w(z) e^{h(z)}=z^{k}(g(z) / d(z)) e^{h(z)}$, where $w(z)=z^{k}(g(z) / d(z))$, $k$ is an integer, $h(z)$ is an entire function, $g(z)$ and $d(z)$ are canonical products (or polynomial) formed respectively with the nonzero zeros and nonzero poles of $f(z)$. Since the poles of $f(z)$ can only occur at the poles of $A(z)$ and $B(z)$, hence $\sigma(d)=\lambda(1 / f) \leq \sigma$.

If $\lambda(f)=\sigma(N(r, 1 / f))<+\infty$, then $\sigma(w)<+\infty$ and

$$
m\left(r, \frac{w^{(j)}}{w}\right)=O(\log r) \quad(j=1,2) .
$$

If $\lambda(f)=+\infty$, then $\sigma(w)=\lambda(f)=\sigma(g)=+\infty . \quad$ By (2.6) and Lemma 6 for any given $\varepsilon>0$, we have

$$
\log T(r, g) \leq r^{\sigma+\varepsilon}, \quad \log T(r, d) \leq(\sigma+\varepsilon) \log r
$$

for sufficiently large $r$, hence

$$
\log T(r, w) \leq M\left\{r^{\sigma+\varepsilon}\right\}
$$

and

$$
m\left(r, w^{(j)} / w\right)=O(\log r T(r, w)) \leq M\left\{r^{\sigma+\varepsilon}\right\} \quad(j=1,2)
$$

for $|z|=r$ outside a set $E_{7} \subset[0,+\infty)$ with finite linear measure, where $M(>0)$ is some constant. 
GROWTH OF SOLUTIONS OF SECOND ORDER LINEAR DIFFERENTIAL EQUATIONS 213

Substituting $f=w e^{h}$ into (1.1), we obtain

$$
-\left(h^{\prime}\right)^{2}=\left(\frac{w^{\prime \prime}}{w}+2 \frac{w^{\prime}}{w} h^{\prime}+h^{\prime \prime}\right)+A\left(\frac{w^{\prime}}{w}+h^{\prime}\right)+B .
$$

Using the similar reasoning as in the proof of Tumura-Clunie's Theorems $[10$, pp. 67-69], we can get

$$
m\left(r, h^{\prime}\right) \leq M_{1}\left\{m\left(r, \frac{w^{\prime}}{w}\right)+m\left(r, \frac{w^{\prime \prime}}{w}\right)+m(r, A)+m(r, B)+S\left(r, h^{\prime}\right)\right\}
$$

for $|z|=r$ outside a set $E_{7}^{\prime} \subset[0,+\infty)$ with finite linear measure, where $M_{1}$ is some positive constant.

By (2.7) (or (2.8)) and (2.10), we know that for $r \notin E_{7} \cup E_{7}^{\prime}(r \rightarrow+\infty)$,

$$
m\left(r, h^{\prime}\right) \leq M_{2}\left(2\left\{r^{\sigma+\varepsilon}\right\}+2 r^{\sigma+\varepsilon}+S\left(r, h^{\prime}\right)\right)
$$

where $M_{2}$ is some positive constant. By Lemma 5, we have

$$
T(r, h)=m(r, h) \leq M_{3}\left(m\left(2 r, h^{\prime}\right)+\log 2 r\right) .
$$

Since Lemma 4 and $S\left(r, h^{\prime}\right)=o\left\{m\left(r, h^{\prime}\right)\right\}$ as $r \rightarrow+\infty$ possibly outside a set of $r$ of finite linear measure, we get $\sigma_{2}(f) \leq \sigma$ from (2.11) and (2.12).

LEMMA $8([4])$. Suppose that $w(z)$ is a meromorphic function with $\sigma(w)=\beta<$ $\infty$. Then for any given $\varepsilon>0$, there is a set $E_{8} \subset(1,+\infty)$ that has finite linear measure and finite logarithmic measure, such that

$$
|w(z)| \leq \exp \left\{r^{\beta+\varepsilon}\right\}
$$

holds for $|z|=r \notin[0,1] \cup E_{8}, r \rightarrow \infty$.

\section{Proof of Theorem 1}

Suppose that $\alpha$ and $\beta$ are real numbers satisfying

$$
\max \{\sigma(A), \lambda(1 / B)\}<\alpha<\beta<\sigma(B) .
$$

If $f(z)$ is a meromorphic solution of $(1.1)$, then by (1.1) it is easy to see that $\sigma(f)=\infty$. By (1.1), we have

$$
|B(z)| \leq\left|\frac{f^{\prime \prime}(z)}{f(z)}\right|+|A(z)|\left|\frac{f^{\prime}(z)}{f(z)}\right| .
$$

By Lemma 1 , there is a set $E_{1} \subset[0,+\infty)$ with a finite linear measure such that for all $z$ satisfying $|z|=r \notin E_{1}$, we have

$$
\left|\frac{f^{(j)}(z)}{f(z)}\right| \leq r[T(2 r, f)]^{3}, \quad(j=1,2) .
$$


By Lemma 8 , there is a set $E_{8} \subset(1,+\infty)$ with a finite linear measure such that for all $z$ satisfying $|z|=r \notin[0,1] \cup E_{8}$, we have

$$
|A(z)| \leq \exp \left\{r^{\alpha}\right\}
$$

On the other hand by Lemma 3, we know that there is a set $H_{3} \subset[0,+\infty)$ with $\overline{\text { dens }} H_{3}=1$ such that

$$
M(r, B) \geq \exp \left\{r^{\beta}\right\}
$$

holds for all $r \in H_{3}$. Now set an $H=H_{3}-\left([0,1] \cup E_{1} \cup E_{5}\right)$, then $\overline{\operatorname{dens}} H=1$. For all $r \in H$, we have by (3.1)-(3.5)

$$
\begin{aligned}
& \exp \left\{r^{\beta}\right\} \leq 2 r \exp \left\{r^{\alpha}\right\}[T(2 r, f)]^{3}, \\
& \exp \left\{(1+o(1)) r^{\beta}\right\} \leq[T(2 r, f)]^{3}
\end{aligned}
$$

as $r \rightarrow+\infty$. Therefore by (3.6), $\sigma_{2}(f) \geq \beta$ holds. Since $\beta$ is arbitrary, we get

$$
\sigma_{2}(f) \geq \sigma(B) \text {. }
$$
$=\sigma(B)$.

Furthermore, if $f(z)$ satisfies (1.3), then by Lemma 7 and (3.7), we get $\sigma_{2}(f)$

\section{Lemmas that are needed for the proof of Theorem 2}

LEMMA 9 ([8]). Let $w(z)$ be a transcendental meromorphic function with $\sigma(w)$ $=\sigma<+\infty$. Let $\Gamma=\left\{\left(k_{1}, j_{1}\right), \ldots,\left(k_{m}, j_{m}\right)\right\}$ denote a finite set of distinct pairs of integers that satisfy $k_{l}>j_{l} \geq 0$ for $i=1, \ldots, m$, and let $\varepsilon>0$ be a given constant. Then there exists a subset $E_{9} \subset(1,+\infty)$ with finite logarithmic measure such that for all $z$ satisfying $|z|=r \notin[0,1] \cup E_{9}$, and $(k, j) \in \Gamma$, we have

$$
\left|w^{(k)}(z) / w^{(j)}(z)\right| \leq r^{(k-j)(\sigma-1+\varepsilon)} .
$$

Also there exists a subset $E_{9}^{\prime} \subset[0,+\infty)$ with finite linear measure such that for all $z$ satisfying $|z|=r \notin E_{9}^{\prime}$, and $(k, j) \in \Gamma$, we have

$$
\left|w^{(k)}(z) / w^{(j)}(z)\right| \leq r^{(k-j)(\sigma+\varepsilon)} .
$$

LEMMA 10. Let $f(z)$ be a meromorphic function with $\lambda(1 / f)<\sigma(f)=\infty$. Then there exists a set $H_{10} \subset[0, \infty)$ with dens $H_{10}=1$ such that for $r \in H_{10}$, there is a point $z_{r}$ satisfying $\left|z_{r}\right|=r$ and

$$
\left|f\left(z_{r}\right) / f^{\prime}\left(z_{r}\right)\right| \leq r .
$$

Proof. Set $\lambda(1 / f)=\alpha<\infty$. We first suppose that $\lambda(f)=\infty$, then

$$
\varlimsup_{r \rightarrow \infty} \frac{\log n(r, 1 / f)}{\log r}=\infty \text {. }
$$


GROWTH OF SOLUTIONS OF SECOND ORDER LINEAR DIFFERENTIAL EQUATIONS 215 There exists $\left\{r_{n}\right\}\left(r_{n} \rightarrow \infty\right)$ satisfying $r_{n} \geq n$ and

$$
n\left(r_{n}, 1 / f\right) \geq r_{n}^{2(\alpha+1)} \geq\left(n r_{n}\right)^{\alpha+1} .
$$

Set a set $H_{10}^{\prime}=\bigcup_{n=1}^{\infty}\left[r_{n}, n r_{n}\right]$. Now we take $\left\{R_{n}\right\}$ such that $n r_{n} / 2 \leq R_{n} \leq n r_{n}$, then

$$
\begin{aligned}
\overline{\operatorname{dens}} H_{10}^{\prime} & =\varlimsup_{r \rightarrow \infty} \frac{m\left(H_{10}^{\prime} \cap[0, r]\right)}{r} \\
& \geq \varlimsup_{n \rightarrow \infty} \frac{m\left\{\left[r_{n}, n r_{n}\right] \cap\left[0, R_{n}\right]\right\}}{R_{n}}=\varlimsup_{n \rightarrow \infty}\left(1-\frac{2}{n}\right)=1 .
\end{aligned}
$$

For $r \in H_{10}^{\prime}$, there is an $n$ such that $r \in\left[r_{n}, n r_{n}\right]$. By (4.3) we get

$$
n\left(r, \frac{1}{f}\right) \geq n\left(r_{n}, \frac{1}{f}\right) \geq r^{\alpha+1} .
$$

On the other hand, for any $\varepsilon(0<3 \varepsilon<1)$, there is $R(>0)$ such that for $r>R$,

$$
r^{\varepsilon-1}<\frac{1}{2}, n(r, f) \leq r^{\alpha+\varepsilon}
$$

hold. Set a set $H_{10}=H_{10}^{\prime} \cap[R,+\infty)$, then $\overline{\operatorname{dens}} H_{10}=1$. For $r \in H_{10}$ by the residue theorem and (4.5), (4.6), we have

$$
\frac{1}{2 \pi} \int_{|z|=r} \frac{f^{\prime}(z)}{f(z)} d z=n\left(r, \frac{1}{f}\right)-n(r, f) \geq r^{\alpha+1}-r^{\alpha+\varepsilon}>\frac{1}{2} r^{\alpha+1} .
$$

So, from (4.7) it is easy to see that there is a point $z_{r}$ with $\left|z_{r}\right|=r$ such that $\left|f^{\prime}\left(z_{r}\right) / f\left(z_{r}\right)\right|>1 / r$. Hence (4.1) holds.

Now we suppose that $\lambda(f)<+\infty$. Then by $\lambda(1 / f)<+\infty, f$ can be expressed in the form $f=w e^{h}$ such that $\sigma(w)=\beta<\infty$ and $h$ is a transcendental entire function. By Lemma 9, we know that there is a set $E_{9}^{\prime} \subset[0,+\infty)$ with linear measure $m E_{9}^{\prime}<\infty$ such that for $|z|=r \notin E_{9}^{\prime}$, we have

$$
\left|\frac{w^{\prime}(z)}{w(z)}\right|<r^{\beta+1} \text {. }
$$

Since $h^{\prime}$ is a transcendental entire function, there is $R(>0)$ such that for $r>R$, we can take a point $z_{r}$ satisfying $\left|z_{r}\right|=r \notin[0, R] \cup E_{9}^{\prime}$ and

$$
\left|h^{\prime}\left(z_{r}\right)\right|=M\left(r, h^{\prime}\right)>r^{\beta+2} \text {. }
$$

By (4.8), (4.9) and

$$
\left|\frac{f^{\prime}(z)}{f(z)}\right| \geq\left|h^{\prime}(z)\right|-\left|\frac{w^{\prime}(z)}{w(z)}\right|
$$

we get 


$$
\left|\frac{f^{\prime}\left(z_{r}\right)}{f\left(z_{r}\right)}\right| \geq \frac{1}{r}
$$

for $r \in[R,+\infty)-E_{9}^{\prime}$. Set a set $H_{10}=[R,+\infty)-E_{9}^{\prime}$, then it is easy to see that $\overline{\text { dens }} H_{10}=1$. Hence (4.1) holds for $r \in H_{10}$.

LEMMA 11 ([3]). Let $g(z)$ be an entire function of order $\sigma$ where $0<\sigma<1 / 2$, and let $\varepsilon>0$ be a given constant. Then there exists a set $H_{11} \subset[0,+\infty)$ with $\overline{\text { dens }} H_{11} \geq 1-2 \sigma$ such that for all $z$ satisfying $|z|=r \in H_{11}$, we have

$$
|g(z)| \geq \exp \left\{r^{\sigma-\varepsilon}\right\}
$$

LEMMA 12. Let $f(z)$ be a meromorphic function with $\lambda(1 / f)<\sigma(f)=\sigma<$ $1 / 2$. Then for $\varepsilon>0$, there exists a set $H_{12} \subset[0, \infty)$ with $\overline{\text { dens }} H_{12}>0$ such that for all $z$ satisfying $|z|=r \in H_{12}$, we have

$$
|f(z)| \geq \exp \left\{(1+o(1)) r^{\sigma-\varepsilon}\right\} .
$$

Proof. Set $f(z)=z^{k} g(z) / d(z)$, where $k$ is an integer, $d(z)$ is a canonical product (or polynomial) formed with the nonzero poles of $f(z), \sigma(d)=\lambda(1 / f)<$ $\sigma(f), g(z)$ is an entire function with $\sigma(g)=\sigma<1 / 2$. By Lemma 11, for a given $\varepsilon(0<3 \varepsilon<\sigma-\lambda(1 / f))$, there exists a set $H_{11} \subset[0,+\infty)$ with $\overline{\text { dens }} H_{11}>0$, such that for all $z$ satisfying $|z|=r \in H_{11},(4.10)$ holds. And there is $R(>0)$ such that

$$
|d(z)| \leq \exp \left\{r^{\lambda(1 / f)+\varepsilon}\right\}
$$

holds for $r>R$ Set an $H_{12}=H_{11} \cap[R,+\infty)$, then $\overline{d e n s} H_{12}>0$. For all $z$ satisfying $|z|=r \in H_{12}$, we get by (4.10) and (4.12)

$$
|f(z)| \geq \exp \left\{r^{\sigma-\varepsilon}-r^{\lambda(1 / f)+\varepsilon}\right\}=\exp \left\{(1+o(1)) r^{\sigma-\varepsilon}\right\} .
$$

LEMMA 13 ([1]). Let $f(z)$ be an entire function of order $\sigma(f)=\sigma<1 / 2$ and denote $A(r)=\inf _{|z|=r} \log |f(z)|, B(r)=\sup _{|z|=r} \log |f(z)| . \quad$ If $\sigma<\alpha<1$, then

$$
\underline{\log d e n s}\{r: A(r)>(\cos \pi \alpha) B(r)\} \geq 1-\frac{\sigma}{\alpha}
$$

where

$$
\underline{\log \operatorname{dens}}(H)=\varliminf_{r \rightarrow \infty}\left(\int_{1}^{r}\left(\chi_{H}(t) / t\right) d t\right) / \log r
$$

and

$$
\overline{\log \operatorname{dens}}(H)=\varlimsup_{r \rightarrow \infty}\left(\int_{1}^{r}\left(\chi_{H}(t) / t\right) d t\right) / \log r
$$

where $\chi_{H}(t)$ is the characteristic function of a set $H$. 
GROWTH OF SOLUTIONS OF SECOND ORDER LINEAR DIFFERENTIAL EQUATIONS 217

LEMMA $14([2])$. Let $f(z)$ be entire with $\mu(f)=\mu<1 / 2$ and $\mu<\sigma=\sigma(f)$. If $\mu \leq \delta<\min (\sigma, 1 / 2)$ and $\delta<\alpha<1 / 2$, then

$$
\overline{\log \operatorname{dens}}\left\{r: A(r)>(\cos \pi \alpha) B(r)>r^{\delta}\right\}>C(\sigma, \delta, \alpha),
$$

where $C(\sigma, \delta, \alpha)$ is a positive constant depending only on $\sigma, \delta$ and $\alpha$.

Lemma 15. Let $A(z), B(z)$ be meromorphic functions such that $B \not \equiv 0$ and

$$
\max \{\sigma(B), \lambda(1 / A)\}<\mu(A) \leq \sigma(A)<\frac{1}{2} .
$$

If $f(\not \equiv 0)$ is a meromorphic solution of the equation $(1.1)$, then $\sigma(f)=\infty$.

Proof. Assume that $f(z)(\not \equiv 0)$ is a meromorphic solution of (1.1), then $f$ is transcendental by $B \not \equiv 0$. Now suppose that $\sigma(f)<\infty$. We set $f(z)=$ $g(z) / d(z)$, where $g(z)$ is an entire function, $d(z)$ is the canonical product (or polynomial) formed with the nonzero poles of $f(z)$. By the fact that the poles of $f(z)$ can only occur at the poles of $A, B$, it follows that $\sigma(d) \leq$ $\max \{\sigma(B), \lambda(1 / A)\}=\beta<\mu(A)$. Since

$$
\begin{gathered}
T\left(r, f^{\prime}\right) \leq 2 T(r, f)+O(\log r), \\
T\left(r, f^{\prime \prime}\right) \leq 2 T\left(r, f^{\prime}\right)+m\left(r, \frac{f^{\prime \prime}}{f^{\prime}}\right) \leq 4 T(r, f)+O(\log r)
\end{gathered}
$$

and

$$
-A=\frac{f^{\prime \prime}}{f^{\prime}}+B \frac{f}{f^{\prime}}
$$

we can get

$$
T(r, A) \leq c T(r, f)+T(r, B)+O(\log r),
$$

where $c$ is a constant. By (4.13) and $\sigma(B) \leq \beta<\mu(A)$, we conclude $\mu(f) \geq$ $\mu(A)$. By $f=g / d$ we have $T(r, f) \leq T(r, g)+T(r, d)+O(1)$. For any given $\varepsilon(0<2 \varepsilon<\mu(f)-\sigma(d)), \quad T(r, f) \geq r^{\mu(f)-\varepsilon}$ and $T(r, d) \leq r^{\sigma(d)+\varepsilon}$ hold for sufficiently large $r$, hence $(1-o(1)) r^{\mu(f)-\varepsilon} \leq T(r, g)+O(1)$, so $\mu(f) \leq \mu(g)$. If $\mu(f)$ $<\mu(g)$, then by $g=f d$, we have

$$
\begin{aligned}
& r^{\mu(g)-\varepsilon_{1}} \leq T(r, f)+T(r, d) \leq T(r, f)+r^{\sigma(d)+\varepsilon_{1}}, \\
& \left(0<2 \varepsilon_{1}<\mu(g)-\mu(f)<\mu(g)-\sigma(d)\right)
\end{aligned}
$$

and

$$
(1-o(1)) r^{\mu(g)-\varepsilon_{1}} \leq T(r, f)
$$

for sufficiently large $r$, hence $\mu(f) \geq \mu(g)$. This is a contradiction. Therefore, $\mu(f)=\mu(g)>\beta$. Similarly, we can get $\sigma(f)=\sigma(g)$. 
From the Wiman-Valiron theory (see $[11,13]$ ), there exists a subset $E \subset(1, \infty)$ with finite logarithmic measure $\operatorname{lm}(E)<\infty$, such that for a point $z$ satisfying $|z|=r \notin E$ and $|g(z)|=M(r, g)$, we have

$$
\frac{g^{\prime}(z)}{g(z)}=\left(\frac{v_{g}(r)}{z}\right)(1+o(1))
$$

where $v_{g}(r)$ denotes the central-index of $g(z)$. By Lemma 9 we know that for any $\varepsilon_{2}\left(0<2 \varepsilon_{2}<\mu(A)-\beta\right)$, there exists a subset $E_{9} \subset(1,+\infty)$ with $\operatorname{lm} E_{9}<\infty$, such that for all $z$ satisfying $|z|=r \notin[0,1] \cup E_{9}$, we have

$$
\left|\frac{d^{\prime}(z)}{d(z)}\right| \leq r^{\beta-1+\varepsilon_{2}} \text {. }
$$

By $\mu(g)=\mu(f) \geq \mu(A)$, there is $R(>1)$ such that

$$
v_{g}(r) \geq r^{\mu(A)-\varepsilon_{2}}
$$

holds for $r>R$. Hence for a point $z$ on which $|g(z)|=M(r, g)$, and $|z|=r \notin$ $[0, R] \cup E \cup E_{9}$, we have by (4.14)

$$
\frac{f^{\prime}(z)}{f(z)}=\frac{g^{\prime}(z)}{g(z)}-\frac{d^{\prime}(z)}{d(z)}=\frac{v_{g}(r)}{z}\left[(1+o(1))-\left(\frac{v_{g}(r)}{z}\right)^{-1} \frac{d^{\prime}(z)}{d(z)}\right] .
$$

By (4.15)-(4.17), we get

$$
\frac{f^{\prime}(z)}{f(z)}=\frac{v_{g}(r)}{z}(1+o(1)) .
$$

By (1.1) and (4.18), we get

$$
-A \frac{v_{g}(r)}{z}(1+o(1))=\frac{f^{\prime \prime}(z)}{f(z)}+B(z) .
$$

Set $A(z)=a(z) / h(z)$, where $a(z)$ is transcendental and $h(z)$ is the canonical product (or polynomial) formed with nonzero poles of $A(z)$. Then $\sigma(h)=\lambda(h)=$ $\lambda(1 / A) \leq \beta, \mu(a)=\mu(A) \leq \sigma(A)=\sigma(a)<1 / 2$. We can choose $\alpha, \delta$ such that

$$
\beta<\alpha<\delta<\mu(A) .
$$

By Lemma 13 (if $\mu(a)=\sigma(a)$ ) or Lemma 14 (if $\mu(a)<\sigma(a)$ ), there exists a subset $H \subset(1, \infty)$ with logarithmic measure $\operatorname{lm} H=\infty$ such that for all $z$ satisfying $|z|=r \in H$, we have

$$
|a(z)| \geq \exp \left\{r^{\delta}\right\} .
$$

By Lemma 8, there exists a set $E_{8} \subset(1, \infty)$ with logarithmic measure $\operatorname{lm} E_{8}<\infty$, such that for all $z$ satisfying $|z|=r \notin[0,1] \cup E_{8}, r \rightarrow \infty$, the following estimations hold:

$$
|B(z)| \leq \exp \left\{r^{\alpha}\right\}, \quad|h(z)| \leq \exp \left\{r^{\alpha}\right\}
$$


GROWTH OF SOLUTIONS OF SECOND ORDER LINEAR DIFFERENTIAL EQUATIONS 219

By Lemma 9, there exists a subset $E_{9} \subset(1, \infty)$ with $\operatorname{lm} E_{9}<\infty$, such that for all $z$ satisfying $|z|=r \notin[0,1] \cup E_{9}$, we have

$$
\left|\frac{f^{\prime \prime}(z)}{f(z)}\right| \leq r^{M}
$$

where $M$ is a constant. It follows from (4.19)-(4.23) that as $r \in H-$ $\left.\left([0, R] \cup E \cup E_{8} \cup E_{9}\right)\left(\operatorname{lm}\left([0, R] \cup E \cup E_{8} \cup E_{9}\right)\right)=\infty\right), r \rightarrow \infty$,

$$
v_{g}(r) \rightarrow 0
$$

holds. But (2.24) contradicts (4.16). Therefore $\sigma(g)=\infty$, i.e. $\sigma(f)=\infty$.

\section{Proof of Theorem 2}

Assume that $f(z)(\not \equiv 0)$ is a meromorphic solution of $(1.1)$, then $\sigma(f)=\infty$ by Lemma 15. We can rewrite (1.1) as

$$
-A(z)=f^{\prime \prime}(z) / f^{\prime}(z)+B(z) f(z) / f^{\prime}(z) .
$$

By Lemma 1 , there is a set $E_{1} \subset[0, \infty)$ with a finite linear measure such that for all $z$ satisfying $|z|=r \notin E_{1}$, we have

$$
\left|f^{\prime \prime}(z) / f^{\prime}(z)\right| \leq r\left[T\left(2 r, f^{\prime}\right)\right]^{2} .
$$

We can choose $\alpha, \rho$ such that

$$
\max \{\sigma(B), \lambda(1 / A)\}=\beta<\alpha<\rho<\sigma(A) .
$$

By Lemma 8 , there is a set $E_{8} \subset[0,+\infty)$ with a finite linear measure such that for all $z$ satisfying $|z|=r \notin E_{8}$,

$$
|B(z)| \leq \exp \left\{r^{\alpha}\right\}
$$

holds. By Lemma 12 , there is a set $H_{12} \subset[0,+\infty)$ with $\overline{d e n s} H_{12}>0$ such that for all $z$ satisfying $|z|=r \in H_{12}$, we have

$$
|A(z)| \geq \exp \left\{(1+o(1)) r^{\rho}\right\} .
$$

Hence by $(5.1)-(5.5)$, for all $z$ satisfying $|z|=r \in H_{12}-\left([0,1] \cup E_{1} \cup E_{8}\right)$ $\left(\overline{\text { dens }}\left\{H_{12}-\left([0,1] \cup E_{1} \cup E_{8}\right)\right\}>0\right)$, we have

$$
\exp \left\{(1+o(1)) r^{\rho}\right\} \leq r\left[T\left(2 r, f^{\prime}\right)\right]^{2}+\exp \left\{r^{\alpha}\right\}\left|\frac{f(z)}{f^{\prime}(z)}\right| .
$$

By Lemma 10, there exists a set $H_{10} \subset[0,+\infty)$ with $\overline{d e n s} H_{10}=1$ such that for $r \in H_{10}$, there is a point $z_{r}$ satisfying $\left|z_{r}\right|=r$ and

$$
\left|f\left(z_{r}\right) / f^{\prime}\left(z_{r}\right)\right| \leq r .
$$

By (5.6) and (5.7), we know that for $r \in H_{10} \cap\left(H_{12}-\left([0,1] \cup E_{1} \cup E_{8}\right)\right)$ 
$\left(\overline{\operatorname{dens}}\left(H_{10} \cap\left(H_{12}-\left([0,1] \cup E_{1} \cup E_{8}\right)\right)\right)>0\right)$, we have

$$
\begin{aligned}
\exp \left\{(1+o(1)) r^{\rho}\right\} & \leq r\left[T\left(2 r, f^{\prime}\right)\right]^{2}+\exp \left\{r^{\alpha}\right\} r \\
& \leq 2 r \exp \left\{r^{\alpha}\right\}\left[T\left(2 r, f^{\prime}\right)\right]^{2}
\end{aligned}
$$

Hence, $\sigma_{2}\left(f^{\prime}\right) \geq \rho$. Since $\rho$ is arbitrary and

$$
T\left(r, f^{\prime}\right) \leq 3 T(r, f)+O(\log r T(r, f))
$$

holds possibly outside a set of $r$ of finite linear measure, combining Lemma 4 we get $\sigma_{2}(f) \geq \sigma(A)$.

Furthermore, if $f$ satisfies (1.4), then by Lemma 7, we have $\sigma_{2}(f) \leq \sigma(A)$. Hence $\sigma_{2}(f)=\sigma(A)$.

\section{Examples}

EXAMPLE 1. The equation

$$
f^{\prime \prime}+\left(2 \cot z-2 z-\frac{1}{z}\right) f^{\prime}-\left(4 z^{2} e^{2 z^{2}}+\left(2 z+\frac{1}{z}\right) \cot z+1\right) f=0
$$

have a solution $f=(1 / \sin z) e^{e^{2^{2}}}$, where $\sigma(B)=2, \sigma(A)=1, \lambda(1 / A)=\lambda(1 / B)=$ 1 , and $\sigma_{2}(f)=2$.

Acknowledgement. The author would like to thank the referee for valuable suggestions to improve my paper.

\section{REFERENCES}

[1] P. D. BarRY, On a theorem of Besicovitch, Quart. J. Math. Oxford Ser. (2), 14 (1963), 293302.

[2] P D. BARRY, Some theorems related to the $\cos \pi \rho$-theorem, Proc. London Math. Soc. (3), 21 (1970), 334-360.

[3] A. Besicovitch, On integral functions of order <1, Math. Ann., 97 (1927), 677-695.

[4] Z.-X. CHEN, The zero, pole and order of meromorphic solutions of differential equations with meromorphic coefficients, Kodal Math. J., 19 (1996), 341-354.

[ 5 ] Z.-X. CHEN AND C.-C. YANG, On the zeros and hyper-order of meromorphic solutions of linear differential equations, Ann. Acad. Sc1. Fenn. Math., 24 (1999), 215-224.

[6] C.-T. Chuang, Sur la comparaison de la crosssance d'une fonction meromorphe et de celle de sa derivee, Bull. Scr. Math., 75 (1951), 171-190.

[7] G. Frank and S. Hellerstein, On the meromorphic solutions of non-homogeneous linear differential equations with polynomial coefficients. Proc. London Math. Soc. (3), 53 (1986), $407-428$.

[8] G. Gundersen, Estimates for the logarithmic derivative of a meromorphic function, plus sımilar estımates, J. London Math. Soc. (2), 37 (1988), 88-104.

[9] G. GUNDERSEN, Finite order solutions of second order linear differential equations, Trans. Amer. Math. Soc., 305 (1988) 415-429. 
[10] W Hayman, Meromorphic Functions, Clarendon Press, Oxford, 1964.

[11] W Hayman, The local growth of power series: a survey of the Wiman-Valiron method, Canad. Math. Bull., 17 (1974), 317-358.

[12] S. Hellerstein, J. Miles and J. Rossi, On the growth of solutions of $f^{\prime \prime}+g f^{\prime}+h f=0$, Ann. Acad. Sci. Fenn. Ser. A I Math., 17 (1992), 343-365.

[13] Y -Z. He AND X.-Z. XIAO, Algebroid Functions and Ordinary Differential Equations, Science Press, 1988 (in Chinese).

[14] G. JANK AND L. VolkmanN, Meromorphe Funktıonen und Differentialgleichungen, Birkhauser, 1985.

[15] K.-H. Kwon, On the growth of entıre functions satısfying second order linear differential equations, Bull. Korean Math. Soc., 33 (1996), 487-496.

[16] K.-H. Kwon, Nonexistence of finite order solutions of certain second order linear differential equations, Koda1 Math. J., 19 (1996), 378-387.

[17] J. K. Langley, Some oscillation theorems for higher order linear differential equations with entire coefficients of small growth, Results Math., 20 (1990), 517-529.

[18] H.-X. YI AND C.-C. YANG, The Uniqueness Theory of Meromorphic Functions, Science Press, Beijing, 1995 (in Chınese).

Department of Mathematics

JiANGXI NORMAL UNIVERSITY

NANChanG, 330027, P.R. ChINA 